\title{
Strong azimuthal combustion instabilities in a spray annular chamber with intermittent partial blow-off
}

\author{
Kevin Prieur ${ }^{1,2, *}$, Daniel Durox ${ }^{1}$, Thierry Schuller ${ }^{1}$, Sébastien Candel ${ }^{1}$ \\ ${ }^{1}$ Laboratoire EM2C, CNRS, CentraleSupélec, Université Paris-Saclay, 92295 Châtenay-Malabry cedex, France \\ ${ }^{2}$ Safran Tech, E\&P, Châteaufort, CS 80112, 78772, Magny-Les-Hameaux, France \\ Email: kevin.prieur@centralesupelec.fr
}

This article reports experiments carried out in the MICCASpray combustor developed at EM2C laboratory. This system comprises 16 swirl spray injectors. Liquid n-heptane is injected by simplex atomizers. The combustion chamber is formed by two cylindrical quartz tubes allowing full optical access to the flame region and it is equipped with twelve pressure sensors recording signals in the plenum and chamber. A high-speed camera provides images of the flames and photomultipliers record the light intensity from different flames. For certain operating conditions, the system exhibits well defined instabilities coupled by the first azimuthal mode of the chamber at a frequency of $750 \mathrm{~Hz}$. These instabilities occur in the form of bursts. Examination of the pressure and the light intensity signals gives access to the acoustic energy source term. Analysis of the phase fluctuations between the two signals is carried out using cross-spectral analysis. At limit cycle, large pressure fluctuations of $5000 \mathrm{~Pa}$ are reached, and these levels persist over a finite period of time. Analysis of the signals using the spin ratio indicates that the standing mode is predominant. Flame dynamics at the pressure anti-nodal line reveals a strong longitudinal pulsation with heat release rate oscillations in phase and increasing linearly with the acoustic pressure for every oscillation levels. At the pressure nodal line, the flames are subjected to large transverse velocity fluctuations leading to a transverse motion of the flames and partial blow-off. Scenarios and modeling elements are developed to interpret these features.

Keywords: combustion dynamics, thermo-acoustic instabilities, annular combustor, swirl flames, spray flames, azimuthal modes, flame blow-off

\section{Introduction}

A considerable research effort has concerned combustion dynamics issues with much of this work focused on problems raised by gas turbines. These machines operate in

\footnotetext{
*Address all correspondence to this author.
}

a lean premixed combustion mode to reduce NOx emissions, the flames are more compact, the power density is augmented and the damping rate is reduced, a combination of factors that promotes instabilities.

In addition, the combustor diameter is in most cases the largest combustor dimension so that instabilities are most often coupled by azimuthal acoustic modes. Under certain conditions this leads to unacceptable levels of pressure oscillations in the system and this has become an essential issue in the design of gas turbine combustion systems. To investigate this class of phenomena it is important to consider annular combustor geometries featuring azimuthal resonances. This has given rise to a variety of analytical investigations including time domain descriptions based on modal expansions and frequency domain representations relying on flame transfer functions or describing functions. Among the more recent works, one may refer to [1-9]. Many models in these articles consider a nonlinear relationship between heat release and pressure fluctuations in the chamber with a saturation at high amplitude $[1,7,10]$. It is generally found that limit cycles coupled to a spinning mode prevail in perfectly axisymmetric systems with intermittent transitions to standing modes due to turbulence [11]. This behavior may be characterized by a spin ratio $[12,13]$. Ghirardo and Juniper [14] introduced additional effects of transverse acoustic disturbances in their model and found that standing modes may prevail for systems featuring large transverse flow oscillations.

Annular system combustion dynamics has also been examined with modern large eddy simulation techniques. Staffelbach et al. [15] carried out an informative simulation of combustion instabilities in an annular chamber demonstrating that when coupling is induced by an azimuthal mode, the flames located near the pressure antinode strongly oscillate in the axial direction while flames located near the pressure nodal line are periodically displaced in the lateral (azimuthal) direction. Simulations reported by Wolf et al. $[16,17]$ showed that phenomena of flashback and liftoff could occur under the effect of large amplitude pressure 
modes and that fluctuations in the heat release rate increased linearly with the level of pressure fluctuations [17].

To validate these theoretical and numerical investigations, it is important to have experimental data at hand and more specifically to examine the dynamics of flames in annular combustor configurations under azimuthal modal coupling. The present article reports such data in the case of a well controlled laboratory scale combustor designated as MICCA-Spray comprising multiple swirling spray injectors and allowing optical access to the combustion region [18].

It is worth examining at this point what has been found in simpler configurations. One may for example simulate the effects of azimuthal waves in annular combustion chambers by considering a linear setup like those used by O'Connor and Lieuwen [19, 20] or by Lespinasse et al. [21]. In both cases, a premixed "V" flame is placed in a transverse acoustic field. At the pressure antinode, the flame is essentially deformed in the axial direction, it exhibits nonlinear features and some lateral ejections of fluid are observed at the injector outlet [22]. At the pressure node, the flames oscillate in the transverse direction and if the flow is swirling, one observes helical perturbations of the reactive front as a result of interaction of the acoustic field with shear regions and with the vortex breakdown process $[19,20]$. The influence of transverse waves acting on the upstream side of the injector unit is analyzed by Hauser et al. [23]. An azimuthal wave established in the plenum of an annular combustor induces a rotational motion of the flames at the injector outlets, associated to asymmetric perturbations of the velocity field in the injector nozzles. The robustness of flames subjected to a transverse acoustic field is investigated by Lespinasse et al. [21]. They showed that gaseous "V" flames located at a pressure node were much more sensitive to blow-off than the same flames placed at a pressure antinode. There are however yet no detailed investigation of the response of spray flames subjected to a transverse acoustic field.

There is a small number of experiments on laboratory scale annular configurations allowing optical access to the combustion region and reproducing, in an idealized fashion, conditions encountered in gas turbines and jet engines [13, 24-28]. In these combustors, spinning or standing modes were observed, usually with transitions between the two types of modes. With premixed flames, anchored on a bluff-body, Dawson and Worth [29] studied the dynamics of the reactive layers depending on their position relative to the azimuthal mode. Although the flames were close to each other, and therefore strongly interacting, flames located near a pressure antinode featured an axial motion, while flames located at a pressure node exhibited a strong lateral motion. To facilitate the observation of standing modes, Worth et al. [30] installed an acoustic forcing system in the combustion chamber to fix the nodal line. In [13, 24, 25, 29], azimuthal instabilities were induced by creating strong interactions between adjacent reactive layers. However other experiments indicate that a small relative spacing between the injectors is not necessarily required to trigger azimuthal instabilities, as shown in studies carried out with matrix burners [26-28] and as evidenced by the present investigation. Additional references dealing with combustion instabilities and interactions with transverse acoustic modes are included in [31].

It is interesting to note that experiments carried out with swirling injectors $[13,25]$, featuring significant pressure losses in the injection unit generally lead to azimuthal instabilities localized in the combustion chamber, while investigations carried out with matrix injectors [26-28], characterized by lower pressure losses indicate that azimuthal instabilities are mainly driven by the plenum. In the annular chamber MICCA equipped with matrix injectors [26-28] instabilities arise over a large domain of operation, whereas with swirling injectors, the system is stable over a broad range of operating points. The unstable areas are narrow $[13,28]$, probably because of the head losses in the injection elements.

This brief review indicates that there are many issues that need further investigation of combustion instabilities coupled by azimuthal modes in annular systems. As all laboratory scale experiments were carried out with premixed flames, it is important to examine cases where the fuel is injected as a spray. Many of the previous studies were conducted with closely packed injectors, and therefore with interacting flames but this does not correspond to the real situation found in industrial combustors. The quantitative evaluation of the flame dynamics with respect to the amplitude of the pressure oscillations is not available but the corresponding data would be of considerable value for theoretical model validation. The levels of oscillations that have been achieved so far in laboratory scale facilities remain modest and it would be important to gather data corresponding to higher pressure amplitudes. The present investigation is intended to deal with the previous issues. Experiments are carried out with liquid fuel spray injection. The annular combustor features combustion instabilities which are mainly of the standing type allowing detailed studies of the dynamics of flames depending on their azimuthal position relative to the nodal line.

This article begins with a brief description of the experimental configuration in section 2. The characterization of a single burner is reported in section 3. Typical instability signals recorded by microphones and a photomultiplier are analyzed in section 4 . The motion of flames located in the vicinity of the pressure antinode and near the pressure nodal line are also considered in this section. When the pressure amplitude reaches high levels it is found that the flames located near the pressure nodal lines may be blown out, a process that may have considerable technical consequences. This point is examined in section 5 and a model is proposed for this mechanism in section 6 .

\section{Experimental set up}

The MICCA-Spray annular system (Fig. 1, left) comprises an annular plenum closed at the top by an annular plate holding sixteen injectors. This constitutes the chamber backplane. The lateral combustor boundaries are formed by two concentric cylindrical walls of different length. These elements allow adapting the impedance of the system to obtain self-sustained instabilities as in $[13,24,25]$. The inner wall is 
made of a quartz tube with a length of $200 \mathrm{~mm}$ and a diameter of $300 \mathrm{~mm}$. The outer wall is made of two parts, a $200 \mathrm{~mm}$ long cylindrical quartz tube and a $400 \mathrm{~mm}$ metal piece. Both pieces share the same inner diameter of $400 \mathrm{~mm}$. Eight channels feed the plenum with pure air injected at ambient temperature. The flow is set in a rotating motion in the injector by a tangential swirler with six $4.5 \mathrm{~mm}$ diameter holes. The injector is terminated by a convergent end piece with an exit diameter $d=8 \mathrm{~mm}$. More details are given in [18]. Liquid $\mathrm{n}$-heptane is injected in the chamber by a simplex atomizer with an orifice placed on the downstream side of the swirler, $6 \mathrm{~mm}$ in recess with respect to the injector outlet. This injector establishes a hollow cone spray in the chamber. In the present experiments n-heptane fuel is chosen for its high volatility. Heavier fuel like dodecane or even kerosene could be used in future studies. Ignition is initiated by a spark plug positioned approximately at $10 \mathrm{~mm}$ from the center of one injector. The position of the spark plug remains roughly the same in all experiments.

The system is equipped with twelve microphones and a photomultiplier (Figure 1, middle). This optical sensor called PM in the following and equipped with an $\mathrm{OH}^{*}$ filter can be placed around the annulus to follow the dynamics of one flame using a spatial filter. It provides a measure of the light intensity radiated by excited hydroxyl radicals. In the case of premixed flames, this signal can be interpreted as a measure of the heat release rate. The case at hand is not premixed but we will nevertheless assume that the PM signal intensity provides to some extent an indication on the combustion intensity. In this set of experiments, the PM is positioned close to the pressure anti-node. Optical and pressure signals are recorded simultaneously. Images of the combustion region are also recorded by two cameras. One CCD camera provides images of the full annular combustor while the second, a high speed intensified camera, yields closer views of the flames for a detailed analysis of the flame motion. The fast motion of the flame during combustion instabilities is observed with a high speed intensified CMOS camera APXi2 $(512 \times 256 \mathrm{px})$ providing an 8 -bit grey level resolution. This unit was set to record images at a sampling rate of 6000 frames per second, with a shutter duration of $166 \mu \mathrm{s}$. In each test, 49,152 images are recorded. The camera amplifier gain remains constant in all experiments. Acoustic pressure is measured by $1 / 4$ inch Brüel \& Kjäer microphones at a sampling rate $f_{s}=32,768 \mathrm{~Hz}$ and the corresponding data are recorded over a period of $8 \mathrm{~s}$. The signals shown in this article are never filtered. Eight of the microphones (designated as "MC") are used to measure the pressure at the chamber backplane. The pressure taps drilled in the annular chamber base (Fig. 1, middle) are located at equal distance from two injectors and connected to straight metallic waveguides crossing the plenum and terminated by a $25 \mathrm{~m}$ flexible tube closed at its extremity. On each waveguide, a microphone is flush mounted at $170 \mathrm{~mm}$ from the chamber back plane. This distance defines a time lag $\tau_{m-b}=0.46 \mathrm{~ms}$ when the combustion chamber has been running for 15 minutes, and the temperature in the plenum reaches $55^{\circ} \mathrm{C}$. This delay between the chamber and microphone is taken into account to synchronize the acoustic pressure and heat release rate signals with the camera images. Four additional microphones (designated as "MP") are plugged on the plenum. Note that the pressure signals and the flame images can be synchronized using the external signal of the camera.

The MICCA-Spray chamber features sixteen stable flames for $n$-heptane fuel injection over a wide range of operating conditions. The sixteen turbulent flames have a typical "M" shape with no visible mutual interaction. The flames are slightly lifted $8 \mathrm{~mm}$ downstream thanks to the convergent end piece of the injector. Flame shapes in the annulus are shown in Fig. 1, right. Whatever the effort made, it was not possible to obtain perfectly symmetric and uniformly balanced injectors due to the small differences in pressure loss induced by the sixteen atomizers. Wall temperature effects can be seen in Fig. 1 with a photograph taken just after the ignition (top) and after 10 minutes of operation (bottom). The MICCA-Spray chamber can be operated over a broad range of conditions but instabilities are found for specific injection parameters. Experiments are then carried out for a global equivalence ratio which ranges from $\phi=0.8$ to 0.9 . The power of the chamber varies from $\mathcal{P}=105$ to $120 \mathrm{~kW}$ and the bulk velocity calculated with the injector diameter $D=$ $8 \mathrm{~mm}$ ranges from $U_{b}=45$ to $50 \mathrm{~m} \mathrm{~s}^{-1}$. The corresponding Reynolds number based on the injector exit diameter varies from 25,800 to 28,700 .

It is interesting to quickly estimate the resonant frequency of the annular combustor at which self-sustained instabilities could be triggered. The chamber has a perimeter $P=2 \pi R \simeq 1.1 \mathrm{~m}$ and a length $l=200 \mathrm{~mm}$. The backplane of the chamber is considered as a solid wall and the top of the chamber is an open end. We make the hypothesis of a mean temperature of $1300 \mathrm{~K}$ equivalent to a global speed of sound $c=720 \mathrm{~ms}^{-1}$ in the entire chamber. The first resonant frequency $f$ can be estimated as follows $f=$ $\left[(c / P)^{2}+(c / 4 l)^{2}\right]^{1 / 2}=746 \mathrm{~Hz}$. This frequency is close to that observed experimentally as it will be shown in the following.

\section{Single burner characterization}

A single injector is placed in a cylindrical burner in order to characterize the aerodynamic and spray structures with laser optical tools. This setup represents a sector of the MICCA-Spray annular chamber. The surface area of the single burner chamber is chosen to correspond to the confinement of a flame in MICCA-Spray. The flame in the single burner is similar in shape to those prevailing in the annular chamber. The burner is equipped with a $70 \mathrm{~mm}$ diameter and $200 \mathrm{~mm}$ long quartz tube. The total power of the single burner is close to $P=7 \mathrm{~kW}$. A 9-bar pressurized fuel tank supplies the atomizer with n-heptane. An argon-ion laser at $514.5 \mathrm{~nm}$ is used to create vertical tomographic slices of the droplet spray under hot fire conditions. The laser slice in Fig. 2 shows the hollow liquid spray cone established in the chamber. Note that droplets are still present all along the combustion zone. This confirms the non-premixed two- 
phase character of the flame. In this image, some blue luminosity indicates that the flame develops from 8 to $30 \mathrm{~mm}$ from the chamber backplane.

The velocity profiles of the air flow and the n-heptane droplet diameters in the single burner are determined with a Phase Doppler Anemometer (PDA) under cold flow conditions. Measurements are carried out without confinement to avoid laser beam deflections due to wall wetting and to the cylindrical shape of the quartz. Measurements with confinement under hot fire conditions confirm that this procedure is adequate. Figure 3 displays the three velocity profiles of the air flow $\left\{U_{r}, U_{\theta}, U_{z}\right\}$ measured at $z=2.5 \mathrm{~mm}$ above the injector outlet for nominal injection conditions, where the chamber is the most unstable. No fuel is injected at this stage and LDA measurements are based on seeding oil droplets (diameter $\left.d_{10}<2 \mu \mathrm{m}\right)$ giving access to the entire air dynamics. Mean data are shown for 50,000 oil droplets to ensure statistical convergence. High axial velocities are observed with a peak $U_{z} \simeq 55 \mathrm{~m} \mathrm{~s}^{-1}$. A well defined recirculation zone is present in the center of the chamber. The air flow also features a sizable azimuthal component. The measured velocity profiles used to determine the swirl number provide a value $S=0.63$.

Figure 4 shows the diameter profiles of the n-heptane droplets injected by the liquid atomizer within the swirling air flow field shown in Fig. 3. Measurements are carried out at $z=2.5 \mathrm{~mm}$ from the injector outlet. At $r=5 \mathrm{~mm}$, i.e. in the dense zone of the spray (see Fig. 2), the mean diameter $d_{10}$ is $\simeq 8 \mu \mathrm{m}$. At the same location, the Sauter Mean Diameter $(\mathrm{SMD}) d_{32}$ is $\simeq 30 \mu \mathrm{m}$. These relatively small diameters confirm the good atomization provided by the liquid fuel injector.

It is worth estimating the vaporization time $\tau_{v}$ corresponding to these droplet diameters. This can be based on the $\mathrm{d}^{2}$ law [32]:

$$
\begin{aligned}
\tau_{v} & \simeq \frac{1}{8} \frac{c_{p}^{g}}{\lambda_{g}} \rho_{l} d^{2} \frac{1}{\ln \left(1+B_{T}\right)} \\
\lambda_{g} & =\lambda_{g 0}\left(1+0.276 \operatorname{Re}^{1 / 2} \mathrm{Sc}^{1 / 3}\right)
\end{aligned}
$$

where $c_{p}^{g}, \lambda_{g 0}$ are the specific heat capacity and heat conductivity of the gas and $\rho_{l}$ and $d$ the liquid density and droplet diameter, and Re and Sc are respectively the Reynolds and Schmidt numbers. The Spalding transfer number $B_{T}=$ $c_{p}^{g}\left(T_{g}-T_{l}\right) / L_{v}$ is here expressed in terms of the gas $T_{g}$ and liquid $T_{l}$ temperatures and latent heat $L_{v}$. These expressions account for convective effects by making use of an effective heat conductivity $\lambda_{g}$ appearing in Eq. (2).

It is standard to evaluate the gas properties by the $1 / 3$ $2 / 3$ rule $\bar{T}=(1 / 3) T_{g}+(2 / 3) T_{l}$. Figure 5 shows vaporization time estimates $\tau_{v}$ and distances of vaporization $l_{v}$ for different gas temperatures $T_{g}$. The reference temperature $T_{l}=$ $300 \mathrm{~K}$ is kept constant. Assuming that the gas and liquid temperatures are $T_{g}=1000 \mathrm{~K}, T_{l}=300 \mathrm{~K}$ and that the velocity differential between the droplets and flow is small, one obtains $\tau_{v} \simeq 0.18 \mathrm{~ms}$ for droplets with a diameter of $10 \mu \mathrm{m}$ and a value four times larger for droplets with a diameter of $20 \mu \mathrm{m}$. Considering the latter droplets, their vaporization delay combined with the chemical time yields a characteristic combustion delay $\tau_{c d}=\tau_{v}+\tau_{c}$ which may be estimated to be about $0.5 \mathrm{~ms}$. It will be seen that this delay is of the order of one half acoustic period $\tau_{c d} \simeq T / 2$, a condition that often leads to an unstable behavior.

\section{Analysis of the self-sustained instabilities}

The MICCA-Spray combustor features self-sustained instabilities with acoustic peak pressure levels of up to $3500 \mathrm{~Pa}$ for a broad range of operating conditions. For specific injection conditions, the peak level may reach up to $6000 \mathrm{~Pa}$ and this then triggers partial blow-off of the annular combustor. This point is examined in section 5 .

\section{Influence of the wall temperature}

The analysis of combustion instabilities is carried out on the annular combustor with hot walls, as typified at the bottom in Fig. 1, right. The self triggering of the instabilities depends on the wall temperature. Figure 6 shows the acoustic pressure in the chamber during $8 \mathrm{~s}$ records corresponding to different wall temperature conditions. The temperature is measured using a surface K-thermocouple placed on the external side of the outer quartz tube, between two injectors, $40 \mathrm{~mm}$ above the back of the chamber. While no pressure fluctuations can be seen when the chamber walls have a temperature under $800 \mathrm{~K}$, one finds that, as soon as the temperature reaches $830 \mathrm{~K}$, instabilities are self triggered. The maximum acoustic level in the chamber increases until the wall temperature is stabilized around $900 \mathrm{~K}$ after 10 minutes of operation. In the next experiments, wall temperature equilibrium is reached before the data acquisition.

The top plots in Fig. 7 show a typical record of the acoustic pressure in the chamber and in the plenum. In this test, the acoustic pressure reaches peak values of $3500 \mathrm{~Pa}$ in the chamber and peak values of $500 \mathrm{~Pa}$ in the plenum. The modes triggered in this annular combustor with swirled spray injectors are mainly chamber modes as in previous experiments carried out with premixed propane and air [13]. The frequency of oscillation when the mode is established is around $750 \mathrm{~Hz}$ (see Fig. 9).

The spin ratio $s$, defined in [12] and modified in [13], is a dimensionless number that characterizes azimuthal modes. This ratio is obtained by decomposing the acoustic pressure in two waves spinning in the counterclockwise and clockwise directions with complex amplitudes $a$ and $b$ and can then be expressed as:

$$
s=\frac{|a|-|b|}{|a|+|b|}
$$

It is used to determine if the mode is standing $(s=0)$ or spinning in the counterclockwise direction $(s=+1)$ or in the clockwise direction $(s=-1)$. In the studied sequence, the standing mode prevails but a spinning mode is triggered 
for a short period of time near $t=6000 \mathrm{~ms}$. A zoom on the acoustic pressure in the chamber is provided when the mode is standing (top right of Fig. 7) and when the mode is spinning (bottom right of Fig. 7). For the standing mode, microphones MC3 and MC7 are in phase opposition. The amplitude slowly decreases for the other microphones meaning that they tend to be close to the pressure node. This mode adopts a nearly perfect first azimuthal (1A) transverse structure. For the spinning mode (Fig. 7, bottom right), $90^{\circ}$ spaced microphones are in phase quadrature and every signal has the same amplitude. Note that the pressure level for the standing mode is at least 4 times greater than that recorded for the spinning mode.

During the previous sequence, the chamber mode is mainly standing as can be deduced from the spin ratio plot in Fig. 7. The nodal line position is determined at several instants in time. The orientation of this line is determined by reconstructing the acoustic mode in the chamber for a large number of angular positions and determining the angle corresponding to the minimum amplitude. One can see in the plot that the nodal line is naturally fixed at $70^{\circ}$ and does not spin during the entire experiment.

Figure 8 shows the distribution of the spin ratio for 121 different experiments confirming the predominance of the standing mode. One should note that, in a configuration with an even number of injectors like the MICCA-Spray chamber, the nodal line will always lie between two injectors. It is at this point not easy to say why the standing mode is much more probable than the spinning mode but one may propose a few explanations. One possibility could be that the standing mode features a larger growth rate than the spinning mode thus promoting the standing mode. This possibility is demonstrated by calculations [33] carried out by combining the flame describing function with a Helmholtz solver in an analysis of the MICCA combustor dynamics equipped with gaseous laminar matrix injectors. Of course the present configuration differs from that investigated in [33] but the conclusion may hold. Another reason may be linked to large transverse velocity fluctuations as will be revealed later, a feature which has been shown to promote standing patterns [14]. The last possibility is that the system is not fully symmetric because there are small differences between the flames due to small differences in the atomization process that can be responsible for this prevalence of the standing mode as mentioned in [1]. To verify that this break in symmetry is influential, the azimuthal position of the eight injectors neighboring the nodal line were exchanged with the eight injectors neighboring the anti-nodal line. This resulted in a $90^{\circ}$ rotation of the nodal line thus underlining the importance of the injector behavior on the mode shape in annular combustors. The tests confirm that the position of the nodal line is related to the inhomogeneity in the flame repartition.

\section{Frequency shift during mode growth and decay}

Note that instabilities develop in the form of long bursts of 100 to $500 \mathrm{~ms}$ corresponding to $\simeq 75$ to 325 cycles of oscillation in Fig. 7. The occurrence frequency of these bursts is close to $1.5 \mathrm{~Hz}$, no matter the operating conditions or the mean acoustic pressure in the chamber. Three bursts of a signal similar to that plotted in Fig. 7 are analyzed to track the evolution of the frequency during the growth and subsequent decay of the mode. The instability frequency in Fig. 9 is first stable around $800 \mathrm{~Hz}$ when the mode begins to grow. As the mode grows to reach a limit cycle, the frequency drops progressively to $720 \mathrm{~Hz}$. This underlines the nonlinear behavior of the flame dynamics as shown in [34]. The phase between the heat release rate and the acoustic pressure will be investigated in the next section. After this decrease, the frequency stabilizes as the mode is at limit cycle for a short period of time and rapidly starts to decay. At the end of the decay, the frequency increases to get back to approximately $800 \mathrm{~Hz}$. This behavior can then be observed for the next two bursts shown in this Figure. Since the combustion process is mainly coupled by a standing mode, it is natural to observe the flame dynamics at the nodal and anti-nodal lines. As described before, the nodal line is fixed by the injector inhomogeneities. This eases recording the flame behavior at different positions with respect to the nodal line.

\section{Flame dynamics at the anti-nodal line}

The anti-nodal line is where the pressure fluctuations reach their highest value and the azimuthal velocity oscillations are lowest. Flames fluctuate at the frequency of the acoustic mode. Figure 10 shows two flames around this line during a full cycle of oscillations. One can see that the flame motion is purely axial with large heat release rate excursions over a cycle: the brightness of the first $\left(0^{\circ}\right.$ and $\left.90^{\circ}\right)$ and the last $\left(180^{\circ}\right.$ and $\left.270^{\circ}\right)$ two images are radically different. The flames are highly perturbed but this does not seem to change their stability whatever the amplitude of the mode. The flame foot remains roughly the same even when the flame is slightly lifted and not anchored at the the injector lips.

A photomultiplier, located as shown in Fig. 1, records the heat release rate fluctuations $\dot{Q}^{\prime}(t)$ of one flame close to the anti-nodal position. As indicated before, it is assumed that the signal from the photomultiplier is roughly proportional to the combustion intensity therefore to the heat release rate of the flame. This is admittedly and approximation in a situation where reactants are not premixed. Figure 11 shows this signal synchronized with the acoustic pressure $p(t)$ recorded by the closest microphone (MC3). Data are processed to extract the relations between the amplitudes of $\dot{Q}(t)$ and $p(t)$ and the relative phase $\varphi$ between these two signals at the frequency $f$ of oscillation. Three different bursts are displayed in this figure, corresponding to a small time interval in the sequence. For each burst, one can see that the phase between $\dot{Q}(t)$ and $p(t)$ progressively increases from a negative value of -1 rad to about zero, i.e. the two signals are then in phase, before the oscillation amplitude begins to decrease.

The relationship between root-mean-square values of relative heat release rate $\dot{Q}^{\prime} / \overline{\dot{Q}}$ and pressure $p^{\prime}$ fluctuations is plotted in Fig. 12 during different bursts to see if there is 
any saturation of the flame response. For the five bursts of the sequence under investigation, one finds that the trend is quasi-linear and perfectly repeatable. This is in agreement with findings reported in [17] but at variance with the nonlinear amplitude saturation models proposed in [1] and modeled in the form of $\dot{Q}^{\prime}=\alpha p^{\prime}-\beta p^{\prime 3}$. In the present study, the amplitude saturation cannot be invoked to explain the sudden decay of the modal oscillations. At the maximum of the amplitude of the bursts, the signals are almost in phase and the fluctuation of the heat release rate remains important. The Rayleigh source term in the acoustic energy balance equation is maximum when the oscillation is at its peak and when it suddenly begins to decrease.

Different scenarios may be invoked to explain this behavior:

1. The decay of the acoustic pressure during the second part of the burst might tentatively be attributed to a dissipation mechanism that has not yet been identified.

2. Another possibility is that the large frequency shift of the oscillations observed during the growth of the bursts leads to a sudden detuning of resonance, a feature observed in some nonlinear coupled oscillators.

3. An alternative is that saturation of the flame response is not due to their longitudinal motion, but rather due to their transverse response at the other injector locations.

4. Strong oscillations may also destroy the central recirculation zone (CRZ) of the swirling flow leading to important modifications of the flame dynamics and spray atomization, with, as a consequence, a suppression of the instability during some instants.

All these assumptions will be examined in future work.

\section{Flame dynamics at the nodal line}

The second remarkable position in the annulus during a standing mode is at the nodal line where the pressure fluctuations are the lowest and the transverse velocity oscillations are the highest. Figure 13 presents a full cycle of oscillation for three flames close to the nodal line. The nodal line is between flames 14 and 15 . Contrary to the anti-nodal line position, the heat release rate does not seem to fluctuate much over a cycle. But these images underline the lateral displacement of flame 14 and 15 during the cycle of oscillation. Flame 14 is on the left of its center at $0^{\circ}$ and then swings to the right at $180^{\circ}$. This is not easy to visualize in still photographs but there is a helical motion associated to the rotating motion of the air stream. Dynamic Mode Decomposition (DMD) [35] of half of the annular chamber is presented in Fig. 14 to enhance the lateral motion of the flame close to the nodal line. This decomposition, at the frequency of the oscillation $f=732 \mathrm{~Hz}$, clearly shows the axial motion of the flame on the sides (as described in the last section) and the lateral sweeping motion of the flame close to the nodal line. This transverse displacement is particularly important for the flame destabilization as will be shown in section 5 .

An analysis of the behavior of flame 14 is carried out to characterize the lateral sweeping motion when a standing mode is triggered. For that the flame is cropped into two half flames from its symmetry axis. The light emission of each half flame $\dot{Q}_{h}^{\prime}$ is determined at each instant of the video sequence (49,152 images at 6000 frames per second). The RMS of the fluctuating light emission $\dot{Q}_{h}^{\prime}$ is calculated by blocks of 4 periods of oscillation. It is again assumed that the light emission is roughly proportional to the heat release rate, which is only an approximation under conditions that are not premixed. The RMS values are then divided by the respective mean intensity of all half images of the sequence to give the relative light intensity variation $\dot{Q}_{h}^{\prime} / \overline{\dot{Q}_{h}}$. As there are many strong bursts in the processed sequence, the relative light intensity can be plotted versus the RMS level of the pressure signal measured close to the anti-nodal position (MC7). Data are plotted for the two half flames in Fig. 15. Given the small size of each block of images (28 images to have about 4 periods at $750 \mathrm{~Hz}$ ), there is some data scatter, but one can see, on average, a linear evolution of the RMS intensity fluctuation related to the pressure fluctuation. This result indicates that the lateral fluctuations of the flames close the nodal line increase regularly with the instability amplitude.

\section{Flame blow-off under high pressure oscillations}

The phenomenon described in the last section can lead, in certain conditions, to a blow-off of several flames around the nodal line. At some point, the flame sweeping motion becomes so intense that the combustion is disrupted eventually leading to a complete extinction of flames close to the nodal line. Figure 16 shows four successive images of the entire annular combustor when this phenomenon is observed. This happens for a short period of time $(t<100 \mathrm{~ms})$ when the acoustic pressure in the chamber reaches a sufficiently large amplitude.

Figure 17 shows a typical sequence where several blowoff events occur. The acoustic pressure in the plenum exceeds three times a peak value $3000 \mathrm{~Pa}$ and one burst reaches a peak value of up to $5500 \mathrm{~Pa}$. In the plenum, a characteristic waveshape can be observed each time the pressure in the chamber exceeds these values. This phenomenon arises when a flame looses its stable position due to the high level of azimuthal velocity fluctuations. This corresponds to the wave generated when the flame re-ignites after being blownoff. Similar waveforms were observed in the large eddy simulations of the light-round in the MICCA annular combustor [36]. At the bottom of Fig. 17, the light intensity recorded for flames 14 and 15 around the nodal line is plotted. One can see that the two flames are entirely blown-off twice during this sequence.

Several experiments with partial blow-off events have been analyzed (not shown here) and used to determine the limit pressure where this process is initiated. Results are plotted in Fig. 18. This estimated limit value is $3000 \mathrm{~Pa}$ peak and this value is repeatably obtained in several experiments corresponding to different operating conditions. This value is of course specific to the injector geometry and to the flame anchoring mechanism. A theoretical approach is proposed 
in the next section, in which a criterion is derived for the occurrence of this partial flame extinction. Observations concerning the blow-off close to the nodal line confirm results reported in [21] about the sensitivity of flames established near a pressure node compared to those located at a pressure anti-node.

\section{Theoretical analysis}

A framework is derived that can be used to interpret the blow-off phenomenon observed for flames located near the pressure nodal line. The data indicate that this phenomenon occurs under high amplitude oscillations. It is also found that the azimuthal velocity induced by the acoustic field displaces the flames in the transverse direction with a direct effect on the heat release rate in the flames located near the pressure nodal line where the transverse velocity reaches its maximum.

One may then consider that it is the displacement amplitude that governs the flame extinction process. When this amplitude is sufficiently large with respect to the size of the central recirculation zone which stabilizes the flame, then the flow is perturbed in a significant manner and the flame is destabilized. The flame displacement induced by the acoustic field can be estimated by $\delta_{\theta} \simeq v_{\theta}^{\prime} / \omega$ where $v_{\theta}^{\prime}$ designates the transverse velocity fluctuation associated with the acoustic mode and $\omega$ denotes the angular frequency of this mode. When this displacement divided by a typical transverse dimension of the Central Recirculation Zone (CRZ) is larger than a certain critical value one may expect a flame blow-off. It is convenient to use the injector diameter $d$ to characterize the size of the CRZ and the previous condition may be cast in the form:

$$
\frac{v_{\theta}^{\prime}}{\omega d}>\psi_{0}
$$

This criterion probably catches much of the physics behind the extinction phenomenon observed in the experiments reported in this article. However, one may consider that this is perhaps not quite enough for the description of this mechanism. One may think for example that the stability margin of the flame is important. If the flame is stabilized but close to blow-off, the situation may be easily perturbed by the external acoustic field. If the stability is assured with a greater margin then it will be less easy to perturb the process. It is then natural to perform a dimensional analysis of this situation. This begins by listing parameters governing the process. In addition to $v_{\theta}^{\prime}, \omega$ and $d$ one may consider that the typical mean injection velocity $U_{b}$ and a typical combustion delay time $\tau_{c d}$ also intervene. It is also known that the swirl number $S$ controls the aerodynamics of the flow. There are six parameters and two independent variables. One may then write the blow-off condition in a new form:

$$
\frac{v_{\theta}^{\prime}}{\omega d}>\psi_{1}\left(\frac{v_{\theta}^{\prime}}{U_{b}}, \frac{U_{b} \tau_{c d}}{d}, S\right)
$$

In the previous expression $b=U_{b} \tau_{c d} / d$ defines the stability of the flame and one may note that $v_{\theta}^{\prime} / U_{b}$ has to be sufficiently large if the acoustic field is to play a role and induce flame blow-off. This can be used to tentatively write the previous expression in the form :

$$
\left(\frac{v_{\theta}^{\prime}}{\omega d}\right)\left(\frac{v_{\theta}^{\prime}}{U_{b}}\right)>\psi_{2}\left(\frac{U_{b} \tau_{c d}}{d}, S\right)
$$

Let us assume that in the absence of perturbation the flame is stable if $b=U_{b} \tau_{c d} / d$ is less than a critical value $b_{*}$. Then, the stability margin may be estimated by the difference between this critical value and the actual value of the stability number: $b_{*}-b$. One may then tentatively propose a blowoff condition in the form :

$$
\left(\frac{v_{\theta}^{\prime}}{\omega d}\right)\left(\frac{v_{\theta}^{\prime}}{U_{b}}\right)>\psi_{3}(S)\left(b_{*}-b\right), \quad \text { where } \quad b=\frac{U_{b} \tau_{c d}}{d}
$$

This criterion takes the form of set of hyperbolic lines shown in Fig. 19. This diagram indicates that flame blow-off will occur if the two parameters $v_{\theta}^{\prime} / \omega d$ and $v_{\theta}^{\prime} / U_{b}$ have sufficiently large values to exceed the product of some function $\psi_{3}(S)$ times the stability margin estimated by $b_{*}-b$. The present experimental data cannot be used to confirm this criterion but they comply with the conclusion that $v_{\theta}^{\prime} / \omega d$ and $v_{\theta}^{\prime} / U_{b}$ should be sufficiently large.

\section{Conclusion}

Experiments have been carried out in a laboratory scale system comprising multiple swirling injectors fed with liquid n-heptane and air in an annular combustor operating at atmospheric conditions. This configuration exhibits strong instabilities coupled by a mode featuring a quasi perfect first azimuthal (1A) transverse structure. The instability arises in the form of a sequence of large excursions of pressure followed by rapid decays. The pressure oscillation reaches peak values of the order of $5000 \mathrm{~Pa}$ peak (about $5 \%$ of the chamber pressure). These experiments reveal that high acoustic pressure levels can be reached without any flame interaction. The microphone signals recorded in the chamber indicate that the instability is always coupled by a quasi standing mode. The nodal line is nearly fixed at the same position in the chamber. Despite the cautions taken to homogenize the liquid fuel distribution in the chamber, it is not possible to suppress some small differences in flow rate and atomization quality. A $90^{\circ}$ interchange of the injectors located near the nodal and antinodal lines turned the nodal line by the same value, indicating that the asymmetries associated with the injection system essentially control the nodal line position. Observations indicate that the unsteady combustion process markedly changes with the position of the flame with respect to the azimuthal mode structure:

- Near the pressure anti-node, where the flames sense maximal axial velocity perturbations from the injector, 
the flame executes a movement in the axial direction with a strong positive heat release rate pulse when the flame extension reaches its maximum value. The relationship between the heat release rate and the pressure oscillation in the chamber has been analyzed in terms of amplitude and phase lag,

- The flames located near the pressure nodal line, submitted to large transverse velocity fluctuation levels are notably displaced in the azimuthal direction. This in turn is reflected in the unsteady heat release rate in opposite parts of these flames. Image processing allowed to determine the evolution of the lateral flame sweeping motion as a function of the amplitude of the pressure fluctuations,

- It is found that the Rayleigh source term always brings energy to the system during the instability bursts. The heat release rate at the anti-nodal line continues to grow linearly with the pressure oscillation and these two quantities are nearly in phase when the amplitude reaches its maximum.

- Saturation and decay that take place at this point might perhaps be due to an additional dissipation in the system, to a sudden detuning of resonance due to a frequency shift or to an alternate flame dynamics saturation mechanisms taking place at the other injector locations. This mechanism requires further investigation.

- When the pressure oscillation amplitude exceeds a certain threshold, the flames located near the pressure nodal line are extinguished, a mechanism that has the immediate effect of reducing the pressure amplitude and allowing re-ignition of the extinguished flames. These local extinctions are accompanied by a strong pressure wave in the combustor and a special signature in the plenum.

A mechanism is proposed for the flame extinction process that defines a critical value for the pressure amplitude. The data reported in this article provide unique indications on the instability process in annular combustors equipped with swirl spray injectors and they can serve to guide modeling and simulations of annular configurations.

\section{Acknowledgements}

This work was supported by Safran Tech, by CNRS and by the French National Research Agency (FASMIC project ANR-16-CE22-0013). The authors wish to thank J. Beaunier and Y. Le Teno for the technical support provided to this research.

\section{References}

[1] Noiray, N., Bothien, M., and Schuermans, B., 2011. "Investigation of azimuthal staging concepts in annular gas turbines". Combustion Theory and Modelling, 15(5), pp. 585-606.

[2] Camporeale, S. M., Fortunato, B., and Campa, G., 2011. "A finite element method for three-dimensional analysis of thermo-acoustic combustion instability". $J$ Eng Gas Turb Power, 133(011506 (13 pages)).

[3] Campa, G., and Camporeale, S. M., 2014. "Prediction of the thermoacoustic combustion instabilities in practical annular combustors". J Eng Gas Turb Power, 136(091504 (10 pages)).

[4] Bauerheim, M., Parmentier, J.-F., Salas, P., Nicoud, F., and Poinsot, T., 2014. "An analytical model for azimuthal thermoacoustic modes in an annular chamber fed by an annular plenum". Combustion and Flame, 161, pp. 1374-1389.

[5] Bauerheim, M., Salas, P., Nicoud, F., and Poinsot, T., 2014. "Symmetry breaking of azimuthal thermoacoustic modes in annular cavities: a theoretical study". Journal of Fluid Mechanics, 760, pp. 431-465.

[6] Ghirardo, G., Juniper, M., and Moeck, J. P., 2015. "Stability criteria forr standing and spinning waves in annular combustors". In Proceedings of ASME Turbo Expo 2015, no. GT2015-43127.

[7] Ghirardo, G., Juniper, M., and Moeck, J. P., 2015. "Weakly nonlinear analysis of thermoacoustic instabilities in annular combustors". Journal of Fluid Mechanics, 805, October, pp. 52-87.

[8] Laera, D., Prieur, K., Durox, D., Schuller, T., Camporeale, S. M., and Candel, S., 2016. "Impact of heat release distribution on the spinning modes of an annular combustor with multiple matrix burners". In Proceedings of ASME Turbo Expo 2016, no. GT2016-56309.

[9] Poinsot, T., 2016. "Prediction and control of combustion instabilities in real engines". In Proceedings of the Combustion Institute, Vol. in press.

[10] Schuermans, B., Paschereit, C. O., and Monkewitz, P., 2006. "Non-linear combustion instabilities in annular gas-turbine combustors". In 44th AIAA Aerospace Sciences Meeting and Exhibit, no. AIAA paper 20060549.

[11] Noiray, N., and Schuermans, B., 2013. "On the dynamic nature of azimuthal thermoacoustic modes in annular gas turbine combustion chambers". Proceedings of the Royal Society A: Mathematical, Physical and Engineering Science, 469(20120535 (15 pages)).

[12] Evesque, S., Polifke, W., and Pankiewitz, C., 2003. "Spinning and azimuthally standing acoustic modes in annular combustors". In AIAA Conference Proceedings Paper 2003-3182.

[13] Bourgouin, J.-F., Durox, D., Moeck, J., Schuller, T., and Candel, S., 2013. "Self-sustained instabilities in an annular combustor coupled by azimuthal acoustic modes". In Proceedings of ASME Turbo Expo 2013, no. GT2013-95010.

[14] Ghirardo, G., and Juniper, M., 2013. "Azimuthal instabilities in annular combustors: standing and spinning modes". Proceedings of the Royal Society A: Mathematical, Physical and Engineering Science, 469(2013032 (16 pages)).

[15] Staffelbach, G., Gicquel, L. Y. M., Boudier, G., and Poinsot, T., 2009. "Large Eddy Simulation of self excited azimuthal modes in annular combustors". Pro- 
ceedings of the Combustion Institute , 32, pp. 29092916.

[16] Wolf, P., Balakrishnan, R., Staffelbach, G., Gicquel, L. M., and Poinsot, T., 2012. "Using LES to Study Reacting Flows and Instabilities in Annular Combustion Chambers". Flow, Turbulence and Combustion , 88(1-2, SI), pp. 191-206.

[17] Wolf, P., Staffelbach, G., Gicquel, L., Müller, J.-D., and Poinsot, T., 2012. "Acoustic and large eddy simulation studies of azimuthal modes in annular combustion chambers". Combustion and Flame, 159, pp. 33983413.

[18] Prieur, K., Durox, D., Beaunier, J., Schuller, T., and Candel, S., 2017. "Ignition dynamics in an annular combustor for liquid spray and premixed gaseous injection". Proceedings of the Combustion Institute, 36(3), pp. 3717-3724.

[19] O'Connor, J., and Lieuwen, T., 2011. "Disturbance field characteristics of a transversely excited burner". Combustion Science and Technology, 183(Issue 5), pp. 427-443.

[20] O'Connor, J., and Lieuwen, T., 2012. "Further characterization of the disturbance field in a transversely excited swirl-stabilized flame". Journal of Engineering for Gas Turbines and Power, 134, p. 011501.

[21] Lespinasse, F., Baillot, F., and Boushaki, T., 2013. "Responses of v-flames placed in an hf transverse acoustic field from a velocity to pressure antinode". Comptes Rendus Mécanique, 341(1), pp. 110 - 120.

[22] Baillot, F., and Lespinasse, F., 2014. "Response of a laminar premixed v-flame to a high-frequency transverse acoustic field". Combustion and Flame, 161(5), pp. $1247-1267$.

[23] Hauser, M., Lorenz, M., and Sattelmayer, T., 2011. "Influence of transversal acoustic excitation of the burner approach flow on the flame structure". J Eng Gas Turb Power, 133, April, p. 041501.

[24] Worth, N. A., and Dawson, J. R., 2013. "Self-excited circumferential instabilities in a model annular gas turbine combustor: Global flame dynamics". Proceedings of the Combustion Institute, 34(2), pp. 3127-3134.

[25] Worth, N. A., and Dawson, J. R., 2013. "Modal dynamics of self-excited azimuthal instabilities in an annular combustion chamber". Combustion and Flame, 160(11), pp. 2476-2489.

[26] Bourgouin, J.-F., Durox, D., Moeck, J. P., Schuller, T., and Candel, S., 2015. "A new pattern of instability observed in an annular combustor: The slanted mode". Proceedings of the Combustion Institute, 35, pp. 32373244.

[27] Bourgouin, J.-F., Durox, D., Moeck, J. P., Schuller, T., and S., C., 2015. "Characterization and modelling of a spinning thermoacoustic instability in an annular combustor equipped with multiple matrix injectors". J Eng Gas Turb Power, 137, p. 021503.

[28] Prieur, K., Durox, D., Schuller, T., and S., C., 2017. "A hysteresis phenomenon leading to spinning or standing azimuthal instabilities in an annular combustor". Com- bustion and Flame, 175, pp. 283-291.

[29] Dawson, J. R., and Worth, N. A., 2014. "Flame dynamics and unsteady heat release rate of self-excited azimuthal modes in an annular combustor". Combustion and Flame, 161(10), pp. 2565 - 2578.

[30] Worth, N. A., Dawson, J. R., Sidey, J. A. M., and Mastorakos, E., 2016. "Azimuthally forced flames in an annular combustor". Proceedings of the Combustion Institute, in press.

[31] O'Connor, J., Acharya, V., and Lieuwen, T., 2015. "Transverse combustion instabilities: Acoustic, fluid mechanic, and flame processes". Progress in Energy and Combustion Science, 49, pp. 1-39.

[32] Ranz, W. E., and Marshall, W. R., 1952. "Evaporation from Drops". Chem. Eng. Prog, 48, pp. 141-146.

[33] Laera, D., Prieur, K., Durox, D., Schuller, T., Camporeale, S. M., and Candel, S., 2016. "Flame describing function analysis of spinning and standing modes in an annular combustor and comparison with experiments". Combustion and Flame, under revision.

[34] Noiray, N., Durox, D., Schuller, T., and Candel, S., 2008. "A unified framework for nonlinear combustion instability analysis based on the flame describing function". Journal of Fluid Mechanics, 615, pp. 139-167.

[35] Schmid, P. J., 2010. "Dynamic mode decomposition of numerical and experimental data”. Journal of Fluid Mechanics, 656, pp. 5-28.

[36] Philip, M., Boileau, M., Vicquelin, R., Schmitt, T., Durox, D., Bourgouin, J. F., and Candel, S., 2014. "Simulation of the Ignition Process in an Annular Multiple-Injector Combustor and Comparison With Experiments". Journal of Engineering for Gas Turbines and Power, 137(3), p. 031501. 
Fig. 1. Left: photograph of the MICCA-Spray annular combustor with sixteen liquid spray injectors. The chamber comprises $200 \mathrm{~mm}$ quartz tubes and a $400 \mathrm{~mm}$ metal cylindrical tube on the outer part of the annulus. The quartz tubes allow full visualization of the flames in the annulus. Middle: Schematic view of the chamber backplane showing the locations of the photomultiplier (PM) and of the different microphones "MCx" in the chamber and "MPx" in the plenum. Flames are numbered from Flame 1 in the clockwise direction. The fixed nodal line position is indicated in red. Right: Direct true-color photograph of the annular chamber showing the influence of the wall temperature. The chamber is under steady operation at a bulk velocity $U_{b}=46 \mathrm{~m} \mathrm{~s}^{-1}$, a global equivalence ratio $\phi=0.86$ and a total power of $\mathcal{P}=113 \mathrm{~kW}$. Top: flame configuration after a few seconds of operation. Bottom: flame configuration after 10 minutes of operation.

Fig. 2. Vertical tomography of the n-heptane droplet spray with flame for $\phi=0.86, U_{b}=46 \mathrm{~ms}^{-1}, \mathcal{P}=7.1 \mathrm{~kW}$ in the single burner setup.

Fig. 3. Mean air velocity profiles $U_{r}, U_{\theta}$ and $U_{z}$ measured at $\mathrm{z}=2.5 \mathrm{~mm}$ for a nominal air flow rate $\dot{m}_{\text {air }}=2.79 \mathrm{~g} \mathrm{~s}^{-1}$ (corresponding to $U_{b}=46 \mathrm{~m} \mathrm{~s}^{-1}$ ).

Fig. 4. n-Heptane droplet diameter profiles $d_{10}$ and $d_{32}$ in cold flow conditions without confinement, $2.5 \mathrm{~mm}$ above the injector outlet for nominal flow rates $\dot{m}_{f u e l}=0.16 \mathrm{~g} \mathrm{~s}^{-1}$ and $\dot{m}_{\text {air }}=2.79 \mathrm{~g} \mathrm{~s}^{-1}$ (corresponding to $U_{b}=46 \mathrm{~m} \mathrm{~s}^{-1}$ ).

Fig. 5. Estimated vaporization times $\tau_{v}$ and vaporization distances $l_{v}$ for different gas temperatures $T_{g}$ and for three droplets diameters $d$. The black dotted line in the second plot corresponds to the distance from the injection point to the quartz wall where n-heptane droplets impact the confinement (approximately $40 \mathrm{~mm}$ ).

Fig. 6. Influence of the wall temperature on the acoustic pressure in the chamber (only microphone "MC7" is plotted). The temperature of the wall is indicated on the top left of each plot. The operation conditions have been kept constant at $\phi=0.85$ and $P=110 \mathrm{~kW}$. 
Fig. 7. Analysis of a sequence with different instability modes in the MICCA-Spray combustor for $\phi=0.86, \mathcal{P}=113 \mathrm{~kW}$. Left: From top to bottom, acoustic pressure in the chamber and in the plenum (locations of the microphones "MPx" and "MCx" are indicated in Fig. 1, middle), associated spin ratio $s$ and orientation of the nodal line. Right: zoom of the acoustic pressure in the chamber for two types of modes, standing (top) and spinning (bottom).

Fig. 8. Spin ratio distribution calculated over 121 bursts.

Fig. 9. Top: acoustic pressure in the chamber over two different bursts recorded by MC1 (blue) and MC7 (red). Bottom: evolution of the instability frequency during the burst. Calculations are made for sections of $27 \mathrm{~ms}$ of oscillations corresponding to approximately 20 periods with a spectral resolution $\Delta f=1 \mathrm{~Hz}$.

Fig. 10. Light emission of two flames for $\phi=0.84, \mathcal{P}=$ $112 \mathrm{~kW}$ around the anti-nodal line during a full cycle of oscillation. Images are averaged over four cycles. Yellow and dark red respectively correspond to high and low light emission levels.

Fig. 11. Acoustic pressure $p$ and heat release rate $\dot{Q}$ (light intensity) fluctuations for a flame at the anti-nodal position for $\phi=0.85, \mathcal{P}=109 \mathrm{~kW}$. The evolution of the phase between $p$ and $\dot{Q}$ is plotted in the last graph.

Fig. 12. Relationship between $p^{\prime}$ and $\dot{Q}^{\prime} / \bar{Q}$ computed for the different bursts in the sequence. $\dot{Q}^{\prime} / \bar{Q}$ is the relative amplitude of the RMS heat release rate fluctuation and $p^{\prime}$ is the RMS value of the pressure fluctuation.

Fig. 13. Light emission of three flames for $\phi=0.85, \mathcal{P}=$ $113 \mathrm{~kW}$ around the nodal line during a full cycle of oscillation. Images are averaged over four cycles. Yellow corresponds to high light intensity while dark red represents low light emission. The center of the flame is indicated by a white dashed line.

Fig. 14. Lateral and axial flame oscillations enhanced using DMD (false colors) at the frequency of the instability $f=732 \mathrm{~Hz}$ for one cycle. The camera is aligned on flame 14 close to the nodal line (see Fig. 1) and records the flame luminosity of half of the annulus during several periods of oscillation.

Fig. 15. Relative light intensity fluctuations of half flames when the acoustic pressure increases during several instability bursts for $\phi=0.86, \mathcal{P}=113 \mathrm{~kW} . \dot{Q}_{h}^{\prime} / \overline{Q_{h}}$ is the relative amplitude of the RMS heat release rate fluctuation and $p^{\prime}$ is the RMS value of the pressure fluctuation.

Fig. 16. True-color photographs of the annular chamber when six flames are blown-off for $\phi=0.85, \mathcal{P}=111 \mathrm{~kW}$.
Fig. 17. Acoustic pressure in the chamber (top), in the plenum (middle) and the light intensity signal from two flames around the nodal line (bottom) for $\phi=0.81, \mathcal{P}=$ $111 \mathrm{~kW}$.

Fig. 18. Pressure in the chamber when the blow-off appears for eight different experiments. The mean acoustic pressure is indicated with the black line.

Fig. 19. Blow-off limits calculated by assuming that $b_{*}=1$, $\psi_{3}(S)=0.19$. The square symbol corresponds to an operating condition leading to blow-off (a peak value of the pressure equal to $3000 \mathrm{~Pa}$ ). 